\title{
Morphologic Complete Response
}

National Cancer Institute

\section{Source}

National Cancer Institute. Morphologic Complete Response. NCI Thesaurus. Code C123595.

The disappearance of all signs of cancer, supported by morphological analysis. 